\title{
Fatal intrahepatic hemorrhage after nadroparin use for total hip arthroplasty
}

\author{
Francesco Ventura • Alessandro Bonsignore • \\ Francesco De Stefano • Enrico Pizzorno • \\ Cristian Palmiere
}

Accepted: 9 July 2014/ Published online: 10 August 2014

(C) Springer Science+Business Media New York 2014

\begin{abstract}
Low-molecular-weight heparins have become the predominant choice for deep venous thrombosis prophylaxis and treatment. However, their use may cause bleeding complications. Intrahepatic bleeding is exceptional and only very few cases have been described. The authors present a unique case of fatal intrahepatic hematoma complicating nadroparin use in a 65-year-old woman with a hepatic cyst who was admitted to hospital for unilateral total hip arthroplasty. At autopsy, hemoperitoneum (2,000 $\mathrm{ml}$ of blood and clots) was evident. A ruptured subcapsular hematoma involving the right lobe of the liver was observed. The hemorrhage within the cyst induced by the nadroparin use was likely responsible for the subsequent hepatic hematoma, liver rupture, and death. This case highlights the need for pathologists and surgeons to be aware of the possibility of intrahepatic hematoma in patients who have received low-molecular-weight heparins, undergone major surgery and present postoperative hemodynamic instability, especially in those with preoperative diagnosis of hepatic cyst.
\end{abstract}

Keywords Low-molecular-weight heparins - Hip arthroplasty · Hepatic cyst · Intrahepatic hematoma . Autopsy

F. Ventura - A. Bonsignore · F. De Stefano - E. Pizzorno Department of Legal Medicine, University of Genova, Genova, Italy

C. Palmiere $(\square)$

University Center of Legal Medicine (Centre Universitaire

Romand de Médecine Légale), Rue du Bugnon 21,

1011 Lausanne, Switzerland

e-mail: cristian.palmiere@chuv.ch

\section{Introduction}

Low-molecular-weight heparins (LMWHs) are prepared by the enzymatic or chemical depolymerization of standard heparin and have become the predominant choice as first line agents for both deep venous thrombosis (DVT) prophylaxis and treatment. However, LMWH use has been shown to increase bleeding complications [1, 2]. Documented risk factors for LMWH-induced hemorrhage include advanced age, concurrent anti-platelet therapy, high doses, concomitant thrombocytopenia, and impaired renal function [1-4]. LMWH-associated hemorrhages in the absence of any trauma range from lower extremity, abdominal wall, and retroperitoneal hematomas to spontaneous splenic rupture $[1,5,6]$. Spontaneous intrahepatic bleeding is exceptional and only very few cases have been described [7]. Here we present a unique case of fatal intrahepatic hematoma and subsequent spontaneous bleeding from the liver surface complicating nadroparin use in a patient with a hepatic cyst.

\section{Case history}

A 65-year-old Caucasian woman diagnosed as having osteoarthritis of the right hip was admitted to hospital for unilateral total hip arthroplasty. Her past medical history was significant for hypertension and tobacco smoking. She did not have a history of any bleeding disorders, coagulopathies, thrombocytopenia, or diabetes and had never received anticoagulant or anti-platelet medication. All laboratory results were unremarkable during preoperative assessment. The liver was enlarged and a hepatic cyst of the anterior part of the right lobe (diameter $2.8 \mathrm{~cm}$ ) was detected by ultrasound. Preoperatively and postoperatively the patient received nadroparin calcium by subcutaneous 
injection starting $12 \mathrm{~h}$ before surgery at $0.4 \mathrm{ml}(3,800 \mathrm{IU})$ per day. The patient was discharged 3 days later in stable condition with normal laboratory values. On postoperative day 9 the patient complained of severe abdominal pain and developed abdominal distention, hypotension, and tachycardia. She was admitted to the intensive care unit and immediately transfused. There was no apparent source of bleeding at the surgical site. However, in view of the abdominal distension, a bedside ultrasound was performed demonstrating free fluid in the abdomen mostly around the perihepatic area. Her medical status continued to deteriorate despite supportive care and she went into shock. Attempted manual cardiopulmonary resuscitation was unsuccessful and the patient died before laparotomy could be performed.

Postmortem examination was requested by the inquiring authorities. Fresh injection marks due to medical intervention were observed on the upper limbs. At autopsy, no rib or sternum fractures were observed. The pericardial sac was unremarkable with no contusions, effusions or adhesions. No hemorrhages or contusions of the epicardium were found. The heart weighed $315 \mathrm{~g}$ and did not reveal any hypertrophy or dilatation. The myocardium did not exhibit fibrosis or ischemic areas. No lacerations or hemorrhages of the cardiac ventricles were observed. The coronary arteries had a normal anatomic course and revealed mild atheromatous disease without significant stenosis. The mediastinum and the thoracic aorta were unremarkable, with no bruising of the wall. The lungs were relatively edematous, with no pulmonary contusions. Examination of the abdominal cavity showed hemoperitoneum $(2,000 \mathrm{ml}$ of blood and clots). A ruptured sub-capsular hematoma involving the anterior and central parts of the right lobe of the liver was observed (Fig. 1). Minute

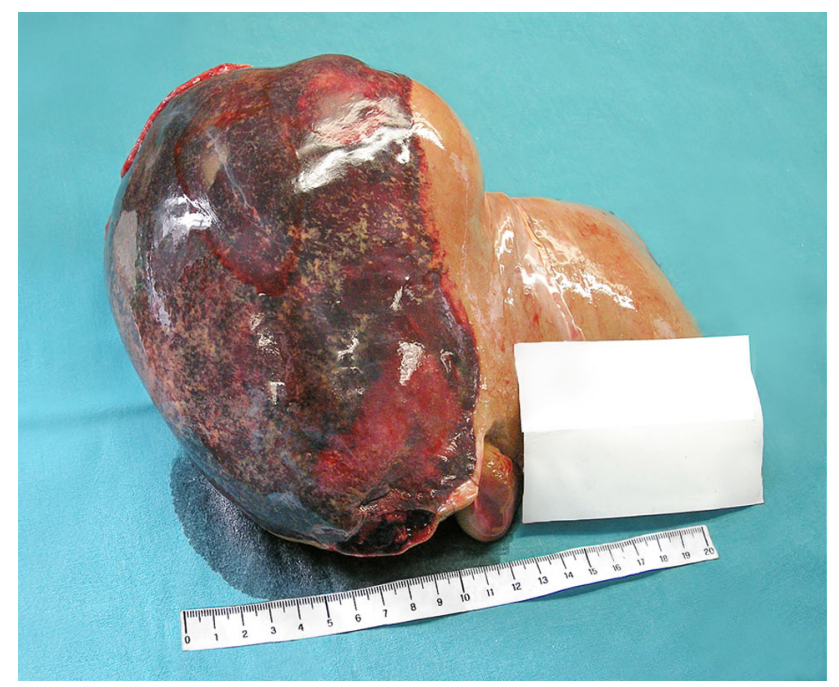

Fig. 1 The hematoma involving the anterior and central parts of the right lobe of the liver remnants of the cyst detected by ultrasound were identified within the hematoma. The spleen and kidneys did not show any significant, macroscopic changes. Retroperitoneal bleeding was not observed. The omentum and small bowel did not show any abnormality. No hemorrhage was noted at the surgical site. The brain weighed $1,050 \mathrm{~g}$ and was macroscopically unremarkable. Sections of most organs were examined microscopically. Histology revealed areas of fatty degeneration of the liver and confirmed the presence of a hemorrhagic, simple cyst of the right lobe. Areas of pulmonary emphysema and hyalinized glomeruli in the kidneys were also observed. Toxicology and bacteriology was unremarkable. Biochemistry failed to reveal results consistent with liver dysfunction.

\section{Discussion}

Deep venous thrombosis is a common, serious complication after major orthopedic surgery of the lower extremities. Because of the high risk of DVT and its considerable morbidity and mortality after abdominal and major orthopedic surgical procedures, the routine use of DVT-prophylaxis is recommended for all patients undergoing hip and knee arthroplasty [2,8].

Heparin is usually given in low doses by subcutaneous injection to prevent venous thrombosis (prophylactic heparin), in higher doses to treat patients with acute venous thromboembolism or with acute coronary syndromes (therapeutic heparin), and in very high doses in patients during open heart surgery [3].

Bleeding is the major complication associated with the use of any type of anticoagulant and thrombolytic therapy and may occur at the injection or instrumentation sites or any remote areas not directly involved with the surgery [2, $3,7]$. Comorbid conditions, particularly recent surgery or trauma, are important risk factors for heparin-induced bleeding. Concomitant anti-platelet medication, thrombolytic therapy, renal failure, and patient age have also been implicated as having a role in hemorrhage subsequent anticoagulant treatment [4].

Hemorrhages associated with anticoagulant use have included abdominal wall hematoma (i.e. rectus muscle sheath hematoma), retroperitoneal hematoma, intrahepatic hemorrhage, spleen rupture, spinal, or epidural hematoma as well as unilateral or bilateral lower extremity hematoma $[1,5,6,9-11]$.

Spontaneous splenic rupture has been reported in association with thrombolytic agents, unfractionated heparin, and LMWHs [9, 12].

Spontaneous retroperitoneal hematomas have been described after warfarin and heparin anticoagulation $[5,6$, 10, 11, 13-15]. Despite the low incidence of severe 
hemorrhage associated with LMWH use, these drugs carry the risk of potentially deadly adverse effects and rare, fatal cases of retroperitoneal hematomas have been observed following anticoagulation with LMWHs [5, 13, 14].

Spontaneous intrahepatic hematomas and hepatic rupture of previously normal livers associated with warfarin, heparin or LMWHs therapy are extraordinary, with no fatal cases reported in the literature [7, 16-19]. Houde and Steinberg [7] reported the case of an 86-year-old woman who was admitted for total hip arthroplasty for osteoarthritis of the right hip. Postoperatively the patients received enoxaparin $30 \mathrm{mg}$ by subcutaneous injection twice a day starting $12 \mathrm{~h}$ after surgery. An intra-hepatic hemorrhage with no capsular rupture was diagnosed on the 3rd postoperative day. Iriz et al. [16] reported the case of a 64-yearold woman who underwent aortic valve replacement due to aortic stenosis. LMWH was not given preoperatively. Dalteparin (10,000 IU/day) was prescribed at the 12th postoperative hour. Free intra-abdominal hemorrhage from a vein near the falciform ligament of the liver and from a bleeding laceration of the splenic capsule occurred on the 2nd postoperative day. More recently, Mir et al. [20] reported the case of a 58-year-old male who was admitted for mitral valve repair due to valve regurgitation. A massive sub-capsular hematoma involving both lobes of the liver and active bleeding was diagnosed $8 \mathrm{~h}$ postoperatively, though the authors did not find a clear association between anticoagulant medication and intra-hepatic hematoma.

LMWHs, including nadroparin, have proven to be as safe and effective as unfractionated heparin for the prophylaxis of DVT in abdominal, hip and knee replacement surgery, with added advantages of decreased laboratory test value monitoring and outpatient drug administration $[5,7$, 13].

Data obtained from meta-analyses and clinical trials comparing LMWH with unfractionated heparin treatment support the inference that LMWH does not result in an increased risk of major bleeding compared with unfractionated heparin [3].

Simple hepatic cyst is a biliary malformation, which does not have communication with the intrahepatic biliary tree. Most cysts measure less than $3 \mathrm{~cm}$ and are asymptomatic. Microscopically, they are lined by a single layer of cuboid or columnar epithelial cells, resembling biliary epithelial cells. In the majority of patients, liver function tests are within the normal range. Asymptomatic, single liver cysts, even when large, do not require treatment or surveillance. However, treatment may be needed if cyst growth leads to symptoms such as abdominal pain, abdominal distension, early satiety, or compression of adjacent structures. Indeed, the cells lining the cavity of hepatic cysts secrete the fluid that causes the cyst to enlarge $[21,22]$.
In the case herein described, the hemorrhage within the hepatic cyst induced by the subcutaneous use of nadroparin was likely responsible for the subsequent hepatic hematoma, liver rupture and death. This sequence of events is even more extraordinary considering that spontaneous hemorrhages of simple hepatic cysts rarely occur [20]. Visceral injury following cardiopulmonary resuscitation (external cardiac massage) has been frequently described in the literature, including cardiac, hepatic, splenic, and mesenteric lacerations. Rare cases of hemoperitoneum have also been reported [23]. In the case reported here, a bedside ultrasound demonstrated the presence of free fluid in the abdomen, mostly around the perihepatic area. Additionally, no fractures of the ribs or sternum were observed at autopsy. The epicardium, myocardium, mediastinum, lungs, aorta, and spleen were unremarkable and did not reveal contusions or lacerations. All these findings tend to exclude the possibility that the hemorrhage within the cyst and the ruptured hematoma of the liver might have been complications of the cardiopulmonary resuscitation and support the hypothesis of a spontaneous intrahepatic hemorrhage.

\section{Key points}

1. Bleeding is the major complication of anticoagulant therapy.

2. Spontaneous intra-hepatic hematoma is an exceptional but potentially lethal condition.

3. Spontaneous retroperitoneal hemorrhage, spleen rupture and spontaneous intra-hepatic hematoma may occur in patients who receive preoperatively and/or postoperatively therapeutic doses of anticoagulants.

4. Surgeons and pathologists must always be aware of the possibility of intra-abdominal bleeding from the liver in patients who have received LMWHs, undergone major surgery and present postoperative hemodynamic instability, especially in those with preoperative diagnosis of hepatic cyst.

\section{References}

1. Daggett JR, Chung S, Smith PD. Bilateral spontaneous, simultaneous lower extremity hematomas in a patient on dalteparin. Int J Surg Case Rep. 2013;4:1080-3.

2. Argun M, Oner M, Saglamoglu M, Karaman I, Guney A, Halici M, Kafadar IH. Fondaparınux versus nadroparın for preventıon of venous thromboembolism after elective hip and knee arthroplasty. Curr Ther Res Clin Exp. 2013;74:49-53.

3. Levine MN, Raskob G, Landefeld S, Kearon C. Hemorrhagic complications of anticoagulant treatment. Chest. 2001;119(1 Suppl):108S-21S.

4. Schulman S, Beyth RJ, Kearon C, Levine MN; American College of Chest Physicians. Hemorrhagic complications of anticoagulant 
and thrombolytic treatment: American College of Chest Physicians Evidence-Based Clinical Practice Guidelines (8th Edition). Chest. 2008;133(6 Suppl):257S-98S.

5. Vayá A, Mira Y, Aznar J, Todolí J, Arguedas J, Solá E. Enoxaparin-related fatal spontaneous retroperitoneal hematoma in the elderly. Thromb Res. 2003;110:69-71.

6. Ernits M, Mohan PS, Fares LG II, Hardy H III. A retroperitoneal bleed induced by enoxaparin therapy. Am Surg. 2005;71:430-3.

7. Houde JP, Steinberg G. Intrahepatic hemorrhage after use of lowmolecular-weight heparin for total hip arthroplasty. J Arthroplast. 1999;14:372-4.

8. White RH, Romano PS, Zhou H, Rodrigo J, Bargar W. Incidence and time course of thromboembolic outcomes following total hip or knee arthroplasty. Arch Intern Med. 1998;158:1525-31.

9. Burg MD, Dallara JJ. Rupture of a previously normal spleen in association with enoxaparin: an unusual cause of shock. J Emerg Med. 2001;20:349-52.

10. Dabney A, Bastani B. Enoxaparin-associated severe retroperitoneal bleeding and abdominal compartment syndrome: a report of two cases. Intensive Care Med. 2001;27:1954-7.

11. Won DY, Kim SD, Park SC, Moon IS, Kim JI. Abdominal compartment syndrome due to spontaneous retroperitoneal hemorrhage in a patient undergoing anticoagulation. Yonsei Med J. 2011;52:358-61.

12. Deprez FC, Colin GC, Hainaut P. Spontaneous (atraumatic) splenic rupture complicating anticoagulant treatment for splenic infarction. Acta Cardiol. 2013;68:421-4.

13. Lissoway J, Booth A. Fatal retroperitoneal hematoma after enoxaparin administration in a patient with paroxysmal atrial flutter. Am J Health Syst Pharm. 2010;67:806-9.
14. Chan-Tack KM. Fatal spontaneous retroperitoneal hematoma secondary to enoxaparin. South Med J. 2003;96:58-60.

15. Sunga KL, Bellolio MF, Gilmore RM, Cabrera D. Spontaneous retroperitoneal hematoma: etiology, characteristics, management, and outcome. J Emerg Med. 2012;43:e157-61.

16. Iriz E, Ereren E, Yuksel O, Kalaycioglu S. Free intra-abdominal hemorrhage after open-heart surgery: an unusual gastrointestinal complication. Tex Heart Inst J. 2006;33:523-5.

17. Karl T. Spontaneous hematoma of the liver associated with heparin therapy. Conn Med. 1983;47:323-5.

18. Roberts MH, Johnston FR. Hepatic rupture from anticoagulant therapy. Arch Surg. 1975;110:1152.

19. Dizadji H, Hammer R, Strzyz B, Weisenberger J. Spontaneous rupture of the liver. A complication of oral anticoagulant therapy. Arch Surg. 1979;114:734-5.

20. Mir NH, Shah MT, Obeid MA, Gallo R, Aliter H. Spontaneous bleeding from liver after open heart surgery. Int J Surg Case Rep. 2013;4:803-4.

21. Yu JH, Du Y, Li Y, Yang HF, Xu XX, Zheng HJ, Li B. Effectiveness of CT-guided sclerotherapy with estimated ethanol concentration for treatment of symptomatic simple hepatic cysts. Clin Res Hepatol Gastroenterol. 2014;38:190-4.

22. Macedo FI. Current management of noninfectious hepatic cystic lesions: a review of the literature. World J Hepatol. 2013;5:462-9.

23. Pinto DC, Haden-Pinneri K, Love JC. Manual and automated cardiopulmonary resuscitation (CPR): a comparison of associated injury patterns. J Forensic Sci. 2013;58:904-9. 